\title{
An Efficient Performance Analysis of Rake Receiver in Spatial Modulation OFDM on a LTE Channel
}

\author{
P.Rajani Kumari ${ }^{1}$, Dr.K.ChennaKesava Reddy ${ }^{2}$, Dr.K.S.Ramesh ${ }^{3}$ \\ ${ }^{1}$ Research Scholar, Department of ECE, Koneru Lakshmaiah Education Foundation, Vaddeswaram, Guntur, \\ A.P, India, rajanitata9@gmail.com \\ ${ }^{2}$.Professor, Stanley College of Engineering \&Technology for Women, Telangana, India \\ kesavary@yahoo.co.in \\ ${ }^{3}$ Professor, Department of ECE, Koneru Lakshmaiah Education Foundation, Vaddeswaram, Guntur, A.P, India \\ dr.ramesh@kluniversity.in
}

\begin{abstract}
Communications in wireless related systems are gathering much attention from the academicians, researching philosophers, and many industrial sectors due to the vital role played by it. Those roles are especially improvising the smooth deployment, cost affordability, and efficiency of the communication towards achieving the practically feasible communication. More specifically, communications in the cellular mode like conventional $3 \mathrm{G}$ and $4 \mathrm{G}$ had improved drastically because of the benefits and usage of it towards the $5 \mathrm{G}$ networks. When anything is being improvised, the number of users using it also augments. Thus, it usually results in the network overloading because of the interferences that the multiple users were causing. Therefore, in this paper, we are objected to propose SM-Spatial Modulation OFDM- Orthogonal Frequency-Division Multi-plexing on the LTE- LongTerm Evolution. We evaluate the proposed SM performance by thorough investigation by BER.
\end{abstract}

Key words: MIMO, OFDM, Rake receiver, Spatial Modulation

\section{INTRODUCTION}

Much security concerns in the transmission operations have been rigorously investigated these days by the demand existing for the rates of it [1],[22]. SM-Spatial Modulation was a novel and out-of-the-box used now by the transmitting operation wherein the symbol from the location of transmit antenna and the individual constellation point in the antenna array conveyed data [2].

Figure 1 shows the typical operation of SM [3]. The source bit-stream is categorized into two blocks, wherein the appropriate symbol and antenna will be chosen [3]. The most crucial benefit of using the SM was that it eliminates the receiving IAI-Inter-Antenna Interference in accordance with the individualistic activation of the

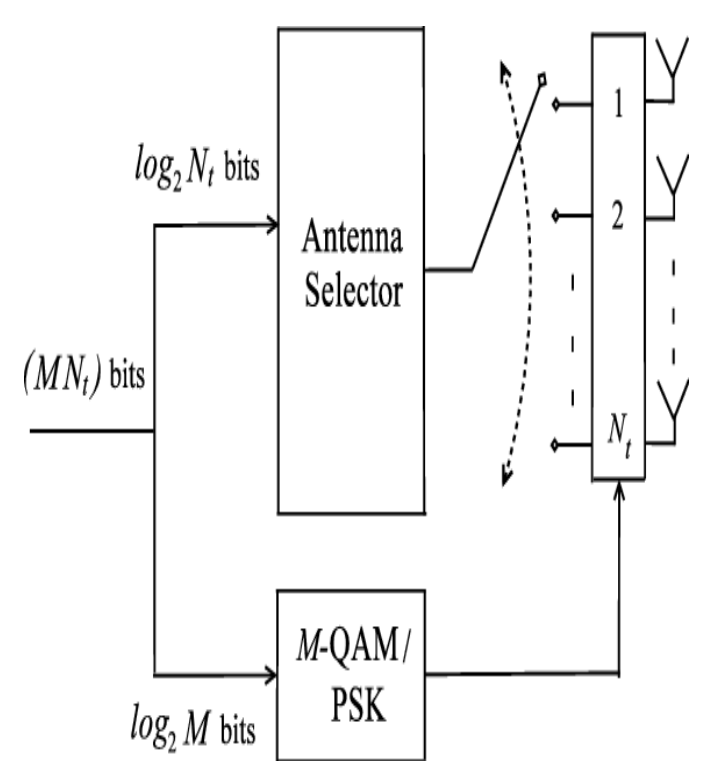

Figure 1: Typical operation of SM [3]

antenna [3]. GSM- Generalized SM was initially implemented with the appropriate type of MLMaximum-Likelihood decoder. But, this type of a decoder was not suitable for actual application in accordance with its exponential complexness, specifically, when the size of constellation or antennas was massive.

m-MCMIMO VLC-massive Multi-Color Multiple-Input Multiple-Output Visible Light Communications [4] were used nowadays for achieving higher-speed and good density- depth coverage towards the next-gen inner data linking. Usage of multiple detecting medium or merely the appropriate combination of filters and detectors could sort out the issues arising due to the overloading and interference occurring in the networks [5]. These improvisations were only possible because of SM operations [5] towards the efforts made in overcoming several technology and infrastructure difficulties posed in many network communications [6]. 
P.Rajani Kumari et al., International Journal of Emerging Trends in Engineering Research, 8(5), May 2020, $1491-1496$

Improvisations in terms of the antenna were also seen towards the optimization of the network parameter impedance [7]. More improvised and better performance-related network contributions were possible by the introduction of the QSM-Quadrature SM [8].

The major objectives of this work are:

- To reduce the overloading by the SM

- To optimize the design and performance by balancing with introduction of rake receiver

- To validate the SM performance with existing literatures

First section of this paper discusses the general intro to the MIMO systems; Second section describes the reported related works concerning the $\mathrm{SM}$ and mobile networks; Third section describes the proposed OFDM SM methodology; Fourth section tells us the performance analysis by taking BER; and Fifth section discusses the drawn conclusion.

\section{RELATED WORKS}

For improvising the transmission operations, [1] was envisioned to suggest security enrichment with the aid of SM-Spatial Modulation as the base. The transmitting and receiving devices were modified to choose the precoding vectors arbitrarily. Their overall view was to make us realize the importance of the receiving end in the mobile network communication.

Usage of SM [9] was not only able to decrease the complexities in the devices and interferences existing between every channel, but it also was able to reduce the consumption of power and some issues of synchronization in the antenna side. Thus, [9] analyzed the effect of the time taken for the switching of antenna against the SM. As far as the comparison was concerned, better relations for the rate of transmission, the efficiency of the spectrum, and ability concerned performance results were established for MIMOMultiple Input Multiple Output systems against the system of the category SIMO-Single Input Multiple Output.

Several trends posed by the VLC-Visible Light Communication was comprehensively surveyed by [10] to make us realize the future views and recent advances in mobile network communications.

Targeting the users of mobile, [11] put frothed the dualdirection based Optical SM for LiFi- Light Fidelity type of cellular networks with the adaptation of SM operation and measuring capable systems. Better outcomes resulted when compared against the traditional Spatial Multiplexing models.
Difficulties caused by SIC-Successive Interface Cancellation were tackled by [12] with the introduction of SM dependent Multi-antenna adopted methodology of NOMA-Non-Orthogonal Multiple Access.

Many surveys were done as in [13]-[14] to discuss the variety of difficulties and achievements of the MIMO with special consideration to SM in the transmission operation in the mobile network communication.

Receiving and transmitting SM operations in $5 \mathrm{G}$ mobile techniques were developed by [15] to improvise the spectral contribution made by the mobile networks. Many conceptual bases of $5 \mathrm{G}$ networks were also seen more often nowadays because of the emergence that the mobile devices have created, especially it was said by [16].

A distributed SM dependent cooperative and variable schema was developed by [17] for the wireless relay networks. Better BER-Bit Error Rate outcomes were resulted due to the fact of achieving higher gain in terms of diversity.

Comprehensive analysis of TCSM-Trellis Coded SM and STBC-SM-Space-Time Block Coded SM along with $\mathrm{SM}$ was done by [2]. A prototype for a RF wave transmission in free space is simulated in[23].

Two of the LR-Lattice Reduction dependent linear type of decoders was utilized by [18] rather than one decoder of the same class since the simulation outcomes were useful only when two were deployed. Good yields of BER were obtained by proceeding with this work.

Despite many out of the box and innovative methods being introduced towards the betterment of the communication in the mobile network, but is still a concern while dealing with the multiple users and complex scenarios of data transmission.

\section{RESEARCH METHOD}

The Typical SM operation was illustrated in figure.1. We will be aiming to avoid the interference with the proposed method comprising the consumers, $\mathrm{C}$ in the MIMO, and OFDM linked systems. Here, the defined consumer will have $\mathrm{M}$ transmitting antennas and $\mathrm{N}$ receive antennas. The overall schema for the proposed method of SM is shown in the below figure. 2. In our proposed method, arbitrary binary data bits assigned to every consumer is given by

$b i^{c}=\left[b i_{1}, b i_{2}, \ldots, b i_{n}\right]$ where inn $c=(1,2, \ldots, C)$

will be initially transmitting it to a Mapper available for SM and every group of $n=\log _{2}(M \times K)$. Here, $\mathrm{K}$ is 


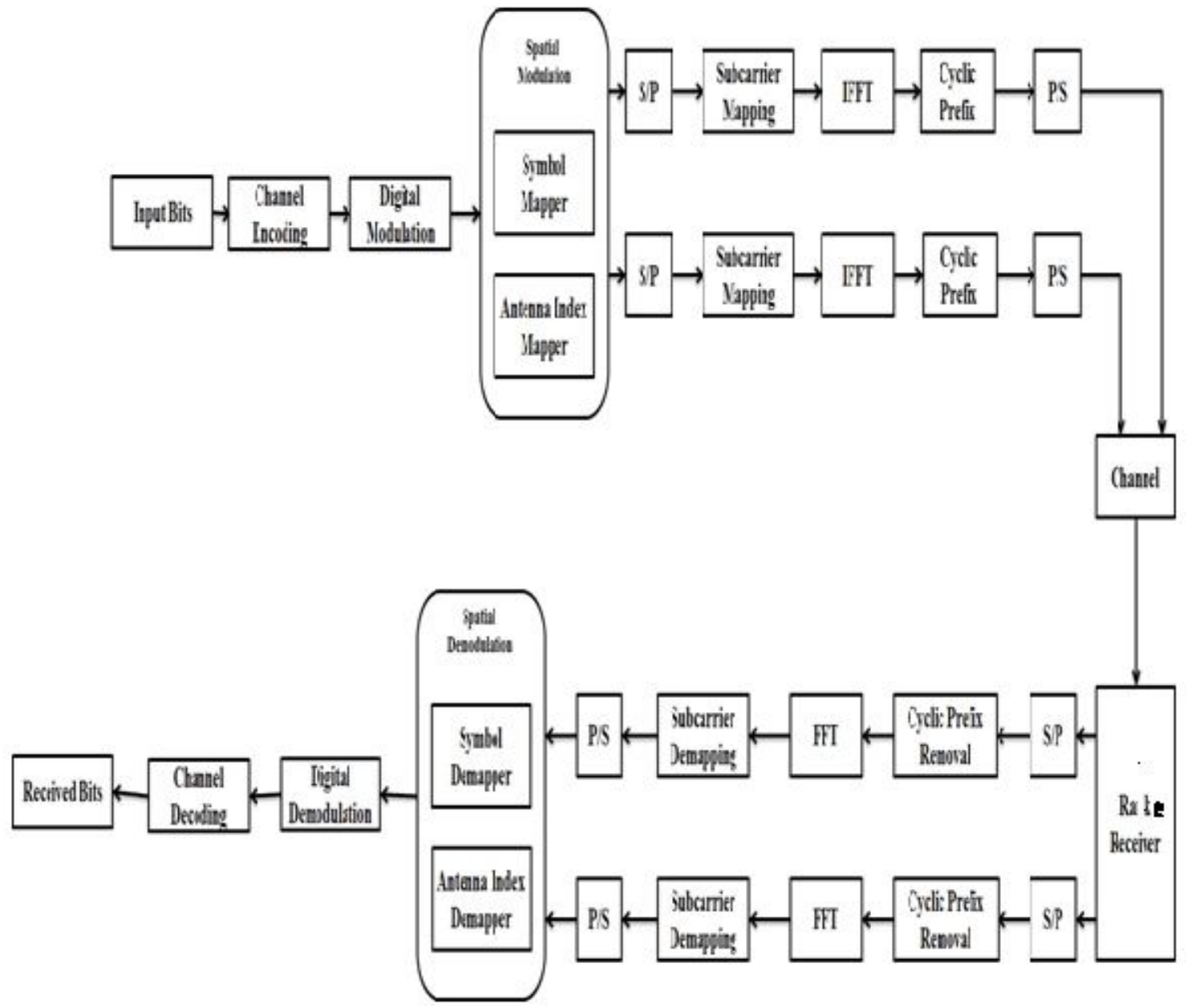

Figure 2: Proposed SM flow

nothing but the QAM-Quadrature Amplitude Modulation constellation size mapped onto a constellation vector,

$$
X^{c}=\left[X_{1}, X_{2}, \ldots, X_{M}\right]^{R}
$$

The restriction of power will be equal to one.

We should realize that every consumer's transmitted bits count will only be based on $\mathrm{K}$ size, as discussed in the previous relation. Then, at the transmitting area, the signals of OFDM- Orthogonal Frequency Division Multiplexing with the aid of IFFT-Inverse Fast Fourier Transform can be given by

$S T D_{i}^{c}(m)=\frac{1}{L^{\frac{1}{2}}} \sum_{0}^{L-1} X_{i}^{c}(k) e^{2 \pi k n j / L}, 0 \leq m \leq L-1$

Where, L: Sub-carrier count in OFDM, $X_{i}^{c}(k)$ : domain info of the frequency at the $i^{\text {th }}$ antenna and $k^{\text {th }}$ subcarriers for the $c^{\text {th }}$ consumer, and $\operatorname{STD}_{i}^{c}(m)$ : signals of the time domain. Commonly, $X^{c}(k)$ constitutes single non 0 elements in every column. The vectors at the output of the OFDM modulator will be executing the transmitting operation from $\mathrm{M}$ antennas at the same time along with the Gaussian noise and MIMO channel. All components of noise are arbitrarily taken as independent Gaussian complexion parameters comprising the variance, $\sigma^{2}$, and mean equal to 0 . Thus, due to the characteristics of the OFDM modulator, the channels in the modulator might show few changes or might be constant against the subcarriers of the frequency domain. $Y^{c}(k)$ will be the size $\left(N^{c} \times L\right)$ of demodulating medium at the output side and every column in the other end on the $m^{\text {th }}$ sub-carrier of the receiving antenna, $N^{c}$, towards the $c^{\text {th }}$ consumer. Right after the above-discussed operation, the information will be allowed to transmit in the module of spatial demodulation, wherein, the data bits will be identified. When this scenario occurs, the difficulty will be posed to antennas doing the receiving, which are related to several consumers who fail to 
achieve coordination intermediary to every other. Thus, interference starts to develop intermediary to them.

Thus, to avoid the occurrence of these interferences, it will be commendable to make use of a few of the precoding schemas. This schema is done by using the block diagonalization and channel inversion appropriately. Here, in this pre-coding schema, the matrix pre-coding gets multiply with every consumer's signal. To make this pre-coding smoother, we will presume that every consumer's receive antennas count will be identical to the transmit antenna count when every consumer in the network has identical receive antenna counts.

First, we will define our proposed work based on MIMO flat categorical faded channels by,

$H_{c}=\left(\begin{array}{ccc}h_{1,1} & \cdots & h_{1, N} \\ \vdots & \ddots & \vdots \\ h_{M, 1} & \cdots & h_{M, N}\end{array}\right)$

Here, $H_{c}: c^{\text {th }}$ consumer's channel of the type MIMO and $h_{i, j}$ : impulsive channel reply to the $i^{\text {th }}$ receiving antenna from the $j^{\text {th }}$ transmitted antenna. This reply will be given by the relation of alike distributed Rayleigh fading as,

$X=\left(\left(p_{1}\right)^{1 / 2} X_{1}\left(p_{2}\right)^{1 / 2} X_{2} \ldots\left(p_{C}\right)^{1 / 2} X_{C}\right)^{R}$,

$\sum_{c=1}^{C} p_{c}=p_{0}$

In the above-indicated relation, $p_{C}: k^{\text {th }}$ data stream transmitting power and $p_{0}$ : overall transmitting power possessed. Improvisations in SNR and smooth splitting could be possible by introducing the rake receiver that generates delays with the help of his inner consisted subreceivers for the sake of retaining the MPCs diversity [19]. On augmenting the correlators count, the receiving performance will be improvised, however, it makes the system to be more complex, big, and makes it cost deficient.

Hence, this makes an exhaustive task whether to go for reducing the complexities in design or improvising the performance. This exhaustive task can be eliminated by utilizing the template signal that re-modifies the received pulses. Afterwards, the outcomes after the multiplication is combined to form a single sample. These both the operations are collectively called as correlations.

Then, we presume that the Generated Rake receiving signal (template signal), $G=\left(R_{1}, R_{2}, \ldots, R_{C}\right)$ as $N \times$ $C$ : an array of pre-coding against every consumer's matrix, $R_{c}$.

$$
\begin{aligned}
y_{c}= & H_{c} \sum_{c=1}^{C} R_{c}\left(p_{C}\right)^{1 / 2} X_{C}=H_{c} R_{c} X_{c}+ \\
& H_{c} \sum_{i=1, i \neq c}^{C} R_{c}\left(p_{C}\right)^{1 / 2} X_{i}+m_{c}
\end{aligned}
$$

\section{RESULTS AND ANALYSIS}

Existing methods of [20] and [21] are used for validating the performance of the proposed SM method. Bit error rate is the ratio of bits or symbols count in the error $\left(C_{S}(e)\right)$ to the overall bits $\left(O_{C}(b)\right)$ in the network. BER is given by,

$B . E . R .=\frac{C_{S}(e)}{O_{C}(b)}$

The indicated figure 3 shows the comparison made between the BER and SNR with the help of the graphical comparison by taking the BER and SNR in y-axis and xaxis respectively.

The proposed categorical SM yields the betwixt outcome in the range of $10^{-1}$ to $10^{-2}$ at the gain being $12 \mathrm{db}$ and $14 \mathrm{db}$ when we take SNR.

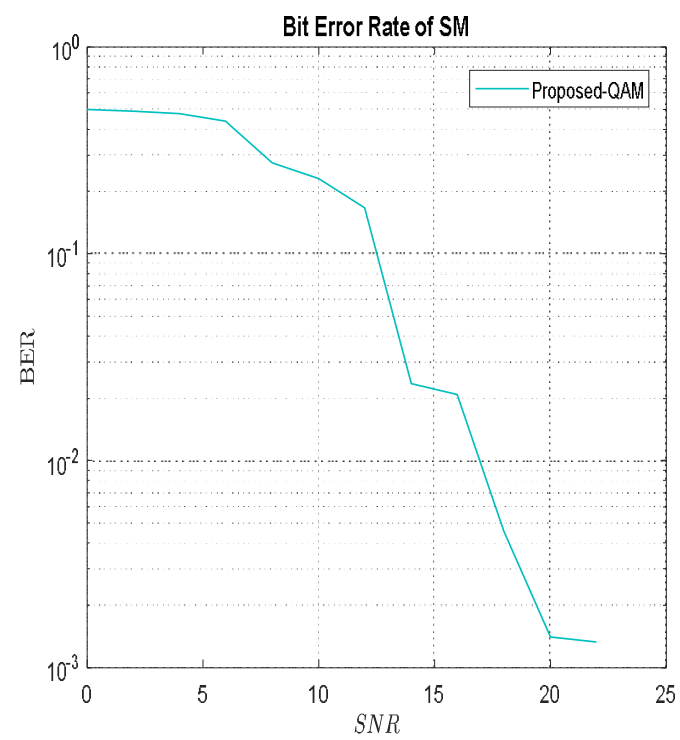

Figure 3: Evaluation of SM performance in terms of BER with SNR

The indicated figure 4 shows the comparison made between the BER and Eb No by plotting the graph between the BER and Eb No in both the axes. The proposed categorical SM yields the betwixt outcome in the range of $10^{-2}$ to $10^{-3}$ at the gain being $9 \mathrm{db}$ and 10 $\mathrm{db}$ while correlating the proposed with all other taken methods. 


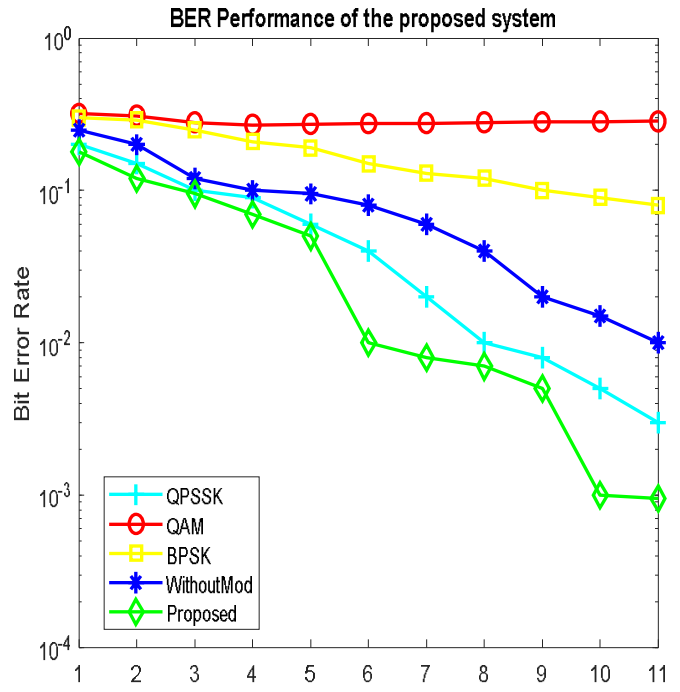

Figure 4: Evaluation of SM performance in terms of BER with Eb No db

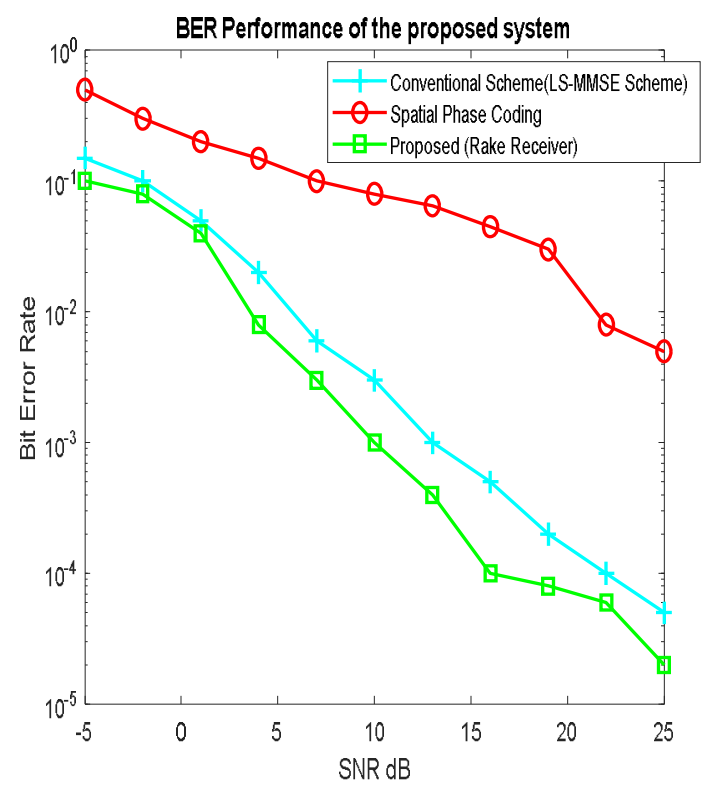

Figure 5: Evaluation of SM performance in terms of BER with SNR with reference to other methods

The indicated figure 5 shows the comparison made between the BER and Eb No by plotting the graph between the BER and Eb No in both the axes.

The devised SM hold good by generating betwixt results in the range of $10^{-3}$ to $10^{-4}$ at the gain being $5 \mathrm{db}$ and $10 \mathrm{db}$ while we compare with all other existing lieratures.

\section{CONCLUSION}

Communications in the cellular mode like conventional $3 \mathrm{G}$ and $4 \mathrm{G}$ had improved drastically because of the benefits and usage of it towards the $5 \mathrm{G}$ networks. But, still many difficulties were realized by the overloading and interferences due to the user's heavier usage these days. We were able to avoid the overloading and contribute much towards the mobile networks. We got the promising results while comparison of BER against some existing literatures as taken in this paper.

\section{REFERENCES}

[1] L. Zhang, M. Sun, Z. Ou, C. Ouyang, and H. Yang, "A Secure Receive Spatial Modulation Scheme Based on Random Precoding," IEEE Access, vol. 7, pp. 122367-122377, 2019. https://doi.org/10.1109/ACCESS.2019.2937962

[2] S. S. Pillai, S. Dhanya, and B. K. Jeemon, "Performance comparison of multicast MIMO systems employing spatial modulation and coded spatial modulation in fading channels," in Proc.International Conference on Intelligent Computing, Instrumentation and Control Technologies (ICICICT), 2017, pp. 1529-1533. https://doi.org/10.1109/ICICICT1.2017.8342798

[3] R. Rajashekar, K. Hari, K. Giridhar, and L. Hanzo, "Performance analysis of antenna selection algorithms in spatial modulation systems with imperfect CSIR," in Proc. 19th European Wireless Conference, 2013, pp. 1-5.

[4] Y. Zhang, Y. Zhu, Y.-Y. Zhang, C. Wang, and Y. $\mathrm{Mu}$, "The Structure Determined of Low Illcondition Structure Constraint Spatial Modulation for Massive Multi-Color MIMO-VLC," in Proc. International Conference on Communications in China (ICCC), 2019, pp. 177-182.

https://doi.org/10.1109/ICCChina.2019.8855888

[5] P. R. Kumari, K. C. K. Reddy, and K. Ramesh, "Hybrid Low Complex near Optimal Detector for Spatial Modulation," International Journal of Electrical and Computer Engineering, vol. 7, p. 818, 2017.

[6] M. Aydemir and K. Cengiz, "Emerging infrastructure and technology challenges in $5 \mathrm{G}$ wireless networks," in Proc. 2nd International Multidisciplinary Conference on Computer and Energy Science (SpliTech), 2017, pp. 1-5.

[7] K. Rachedi, D. T. P. Huy, A. Ourir, and J. de Rosny,"Reconfigurable Split Ring Resonators for Spatial Modulation Communications," in Proc. 13th European Conference on Antennas and Propagation (EuCAP), 2019, pp. 1-5.

[8] S. Adnan, Y. Fu, N. U. R. Junejo, Z. Chen, and H. Esmaiel, "Sparse detection with orthogonal matching pursuit in multiuser uplink quadrature spatial modulation MIMO system," IET Communications, vol. 13, pp. 3472-3478, 2019. https://doi.org/10.1049/iet-com.2019.0321

[9] E. Soujeri and G. Kaddoum, "The impact of antenna switching time on spatial modulation," 
P.Rajani Kumari et al., International Journal of Emerging Trends in Engineering Research, 8(5), May 2020, $1491-1496$

IEEE Wireless Communications Letters, vol. 5, pp. 256-259, 2016.

[10] S. Al-Ahmadi, O. Maraqa, M. Uysal, and S. M. Sait, "Multi-user visible light communications: State-of-the-art and future directions," IEEE Access, vol. 6, pp. 70555-70571, 2018.

[11] M. D. Soltani, M. A. Arfaoui, I. Tavakkolnia, A. Ghrayeb, M. Safari, C. Assi, et al., "Bidirectional optical spatial modulation for mobile users: Towards a practical design for lifi systems," arXiv preprint arXiv:1812.03109, 2018.

[12] C. Zhong, X. Hu, X. Chen, D. W. K. Ng, and Z. Zhang, "Spatial modulation assisted multiantenna non-orthogonal multiple access," IEEE Wireless Communications, vol. 25, pp. 61-67, 2018.

https://doi.org/10.1109/MWC.2018.1700062

[13] N. Agarwal, "Spatial Modulation Technique: Achievements and Challenges," in Proc. Computing, Communication and Signal Processing, ed: Springer, 2019, pp. 441-448.

[14] K. J. Kim, M. Wen, M. Di Renzo, T. A. Tsiftsis, K.-C. Chen, and N. Al-Dhahir, "Guest Editorial Spatial Modulation in Emerging Wireless Systems," IEEE Journal on Selected Areas in Communications, vol. 37, pp. 1945-1948, 2019.

[15] D. Phan-Huy, Y. Kokar, J. Rioult, N. Malhouroux-Gaffet, J.-C. Prevotet, P. Ratajczak, et al., "First Demonstration and Visualization of Receive Spatial Modulation Using the" Radio Wave Display," in Proc. 21st International ITG Workshop on Smart Antennas .2017.

[16] K. Cengiz and M. Aydemir, "Next-Generation Infrastructure and Technology Issues in 5G Systems," journal of communications software and systems, vol.14, 10.24138/jcomss.v14i1.422 2018.

[17] Q.-T. Hoang, "Distributed Spatial Modulation based Cooperative Diversity Scheme," International Journal of Wireless \& Mobile Networks (IJWMN) Vol, vol. 10, 2018. https://doi.org/10.5121/ijwmn.2018.10203

[18] Y. Bao, L. Zhang, R. Xie, and R. RamirezGutierrez, "Low-complexity lattice reduction aided detection for generalised spatial modulation," in proc. International Symposium on Wireless Communication Systems (ISWCS), 2016, pp. 253-257.

[19] V. Goyal and B. Dhaliwal, "Analyzing Pulse Position Modulation Time Hopping UWB in IEEE UWB Channel," Computer Science \& Telecommunications, vol. 46, 2015.

[20] R. Jeya and B. Amutha, "Optimized semiblind sparse channel estimation algorithm for MUMIMO OFDM system," Computer Communications, vol. 146, pp. 103-109, 2019.

[21] M.-J. Paek, W.-C. Kim, M.-Y. Kim, and H.-K. Song, "Spatial Phase Coding With CoMP for Performance Enhancement Based on MIMOOFDM in HetNet System," IEEE Access, vol. 7, pp. 62240-62250, 2019.

https://doi.org/10.1109/ACCESS.2019.2914692
[22] John Martin Ladrido, Jonnel Alejandrino, Emmanuel Trinidad, and Lawrence Materum, "Comparative Survey Of Signal Processing And Artificial Intelligence Based Channel Equalization Techniques And Technologies," International Journal of Emerging Trends in Engineering Research, Vol 7, No. 9. https://doi.org/10.30534/ijeter/2019/14792019.

[23] Aaron Don M. Africa, Lourdes Racielle Bulda, Emmanuel Del Rosario, Matthew Zandrick Marasigan, Isabel Navarro," Radio Wave Propagation: Simulation of Free Space Propagation Path Loss" International Journal of Emerging Trends in Engineering Research, Vol 8, No. 2

https://doi.org/10.30534/ijeter/2020/07822020 